\title{
Biomedical Image Analysis using Region Growing Segmentation Approach
}

\author{
Sangita A. Dubey \\ Research Scholar \\ Faculty of Engineering \\ Pacific Academy of Higher Education and Research \\ University, Udaipur
}

\author{
Vijay R. Rathod, PhD \\ Head, Dept of Electronics \& Telecom \\ St Xaviers Technical Institute Mumbai
}

\begin{abstract}
This paper presents an efficient detection method to highlight the tumors present in brain images. The obtained results further help in finding the size and location of the tumor. The method is applied on brain images having tumors. Using region-growing method we merge the adjacent detected pixels on homogeneity criteria, to obtain the flaws. Region growing starts with seed(s). The seed value is determined with the help of histogram analysis. The peaks and valleys of histogram help in determining the seed value. The developed algorithm is explained and applied on the welding images and some preliminary results are shown which are found encouraging.
\end{abstract}

\section{Keywords}

Image segmentation, region growing, tumor detection, seed, and homogeneity

\section{INTRODUCTION}

Segmentation of an image has always been a key problem in computer vision. Image segmentation is a process in which regions or features sharing similar characteristics are identified and grouped together. Image segmentation is based on thresholding, edge detection, region detection or combination of any of these techniques. The segmentation should separate the regions that are homogenous to the particular criteria chosen for analysis, so that the variations of segmented area should be considerably less than the variation at borders. The segmented area should have smooth shedding and texture. There should not be large variation in homogeneity criteria with in a single segment. Small data must be clear for further analysis and the position of border obtained after segmentation must match with local maxima, ridges and saddle points of local gradient the measurements [1-2].

Segmentation basically divides an image into segments having of the following characteristics.

- Looks uniform

- Belongs to single object

- Have some uniform attributes

- All pixels related to it are connected

Most segmentation techniques are either based on discontinuity or similarity criteria. Region growing is one of the similarity-based techniques.

Region based technique is based on the grouping of the common patterns of intensity values within a cluster of neighboring pixels. The cluster is represented as region. The regions grouped according to their function or anatomical role, are basically based on criteria of homogeneity. So the segmentation is a process of extracting and representing information from an image in the form of group of pixels having homogeneity. Different criteria like texture, colour, gray level etc. can be used, even in combination for regionbased segmentation. Goal of the technique is to determine the regions on the basis of pre-defined homogeneity criteria. Two of the most widely used methods are split and merge and region growing [10-11]

Region growing technique gives good results where borders are difficult to detect and to generate better results in noisy images. We applied the region growing approach for segmentation of weld images. The region growing starts with seed and small areas are merged to obtain a region having all the pixels having predefined criteria. Merging of regions is often based on comparing the difference of their feature measure with a predefined value known as segmentation threshold. An appropriate threshold is crucial to get successful region growing results. In the existing method, the most suitable threshold value is determined by histogram analysis. Although single threshold may be sufficient to segment an image but multiple thresholds give better results in complex image. With the help of iteration the threshold value that gives best results can be obtained easily. Existing algorithms applied for region growing have multiple threshold and these are clubbed together to get best results. It is a position dependent threshold method determined using a prior knowledge about image or by local thresholding techniques applied on different parts of image [12-13]. Local thresholding technique is used on different parts of image to select the most suitable threshold for merging.

\section{REGION GROWING METHODOLOGY}

Region growing is a technique, which helps to extract boundary of an image. In this process the pixels are grouped or sub regions are combined to form large regions, based on predefined criteria. The predefined criteria may be based on different homogeneity properties of an image i.e. intensity value of pixels texture, motion, shape, size, variance and colour. In region growing, the basic idea is to divide an image into zones of maximum homogeneity [14-15].

Basic purpose of region growing is to segment entire image $R$ into smaller sub images $\mathrm{R}_{i}=1,2 \ldots . \mathrm{N}$ which satisfy the following conditions.

$$
\begin{aligned}
& \bigcup_{\mathrm{R}=1}^{N} \mathrm{n}^{i} \quad ; \\
& \mathrm{P}\left(\mathrm{R}^{i}\right)=\text { True } ; \quad \mathrm{i}=1,2 \ldots \ldots . . \mathrm{N} ;
\end{aligned}
$$$$
\text { b) }
$$ 
c)

$$
\mathrm{R}^{i} \cap \mathrm{R}^{j}=\varnothing, \quad \mathrm{i} \neq \mathrm{j}
$$

Where $\mathrm{R}_{i}$ and $\mathrm{R}_{j}$ are adjacent

$$
\text { d) } \mathrm{P}\left(\mathrm{R}_{i} \mathrm{UR} \mathrm{R}_{j}\right)=\text { False, } \mathrm{i} \neq \mathrm{j}
$$

In region growing, an initial set of small areas is iteratively merged according to similar constraints. Starting up with seed point and compare it with neighboring pixels, region is grown from the seed pixel by adding in neighboring pixels that are similar in predefined criteria. The size of the region increases by using 4 adjacent or 8 -adjacent neighbor. When the growth of one region stops, the other seed pixel, which does not belong to any region, is chosen and process of region growing is started again. This whole process is continued until all pixels belong to some region. It gives good result that corresponds well to observed edges. Starting with one seed gives bias results in favors of seed chosen first, so simultaneous region growing technique are used. A number of regions are allowed to grow simultaneously and similar regions will gradually merge with each other.

In region growing adjacency is major control it can be 4 neighbor (adjacent) or 8 neighbor (adjacent), pixel similarity is secondary

\begin{tabular}{|l|l|l|l|l|l|l|l|l|l|l|l|}
\hline 4 & 3 & 2 & 3 & 4 & & & 2 & 2 & 2 & 2 & 2 \\
\hline 3 & 2 & 1 & 2 & 3 & & & 2 & 1 & 1 & 1 & 2 \\
\hline 2 & 1 & 0 & 1 & 2 & & & 2 & 1 & 0 & 1 & 2 \\
\hline 3 & 2 & 1 & 2 & 3 & & & 2 & 1 & 1 & 1 & 2 \\
\hline 4 & 3 & 2 & 3 & 4 & & & 2 & 2 & 2 & 2 & 2 \\
\hline
\end{tabular}

Fig 1: Region Growing Technique

Fig 1.Proximity; define connection between image pixels 4 neighbor (adjacent) or 8 neighbor (adjacent)

Suppose $A=(p 1, q 1), B=(p 2, q 2)$ are two image points;

$$
\begin{aligned}
& \mathrm{D}(\mathrm{A}, \mathrm{B})=\sqrt{(p 1-p 2)^{2}+(q 1-q 2)^{2}} \\
& \mathrm{D}_{4}(\mathrm{~A}, \mathrm{~B})=|p 1-p 2|+|q 1-q 2| \\
& \mathrm{D}_{8}(\mathrm{~A}, \mathrm{~B})=\max \{|p 1-p 2|,|y 1-y 2|\}
\end{aligned}
$$

Region Growing Algorithm

- $\quad$ Start at some location (s) (seeds)

- Propagate from seed or seeds to its neighbor

- Only add adjacent pixels that satisfies with homogeneity criterion of region

- Repeat the Steps from 2 to 3 until it reaches the boundary of the region

- Can grow multiple regions in parallel and in competition [16-17]

\section{RESULTS AND DISCUSSIONS}

In region growing approach the segmentation of welding images start with 'seed' and small areas get merged to obtain a region having all the pixels with pre-defined characteristics. Merging of region is often based on comparing the difference of their feature measure with a predefined value known as segmentation threshold. Threshold determination is difficult. The selection of an appropriate threshold is crucial to get successful region growing results. In the applied algorithm, the threshold value is determined by histogram analysis. The valleys and peaks of histogram help in determining the threshold values. Although single threshold may be sufficient to segment an image but multiple thresholds give better results in complex images. With the help of iteration method, the threshold which gives best result can be obtained easily.

Existing algorithms applied for region growing have multiple thresholds and these results are clubbed together to get best results. It is a position dependent threshold method. Local thresholding technique is used on different parts of image to select the most suitable threshold for merging two regions.

Fig.1 shows a original image having tumor. Tumors are clearly identifiable after the application of algorithm .The thresholded \& segmented image, depicting the tumor is shown in Fig. 2,3,4 \& 5. Also other modality of image is compared here.
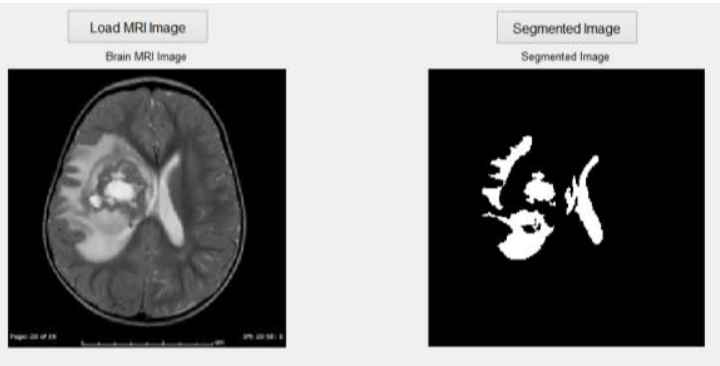

Fig 2: MRI Segmented Image having Tumor at Left Side

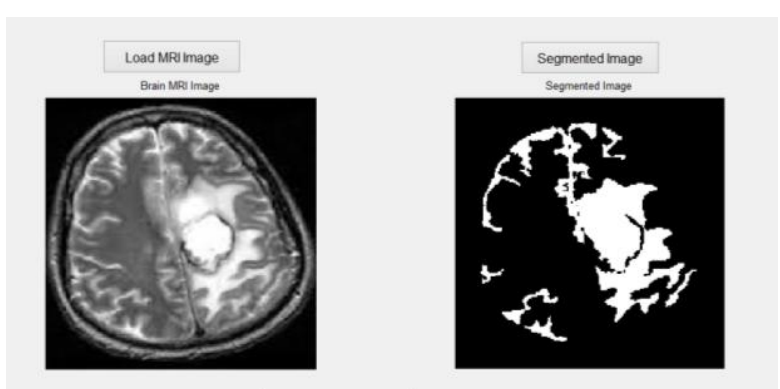

Fig 3: MRI Segmented Image having Tumor at Right Side

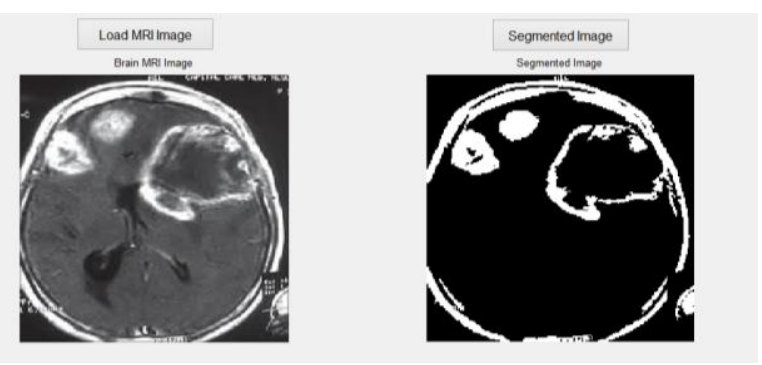

Fig 4 MRI Segmented Image having Tumor at Right Side 


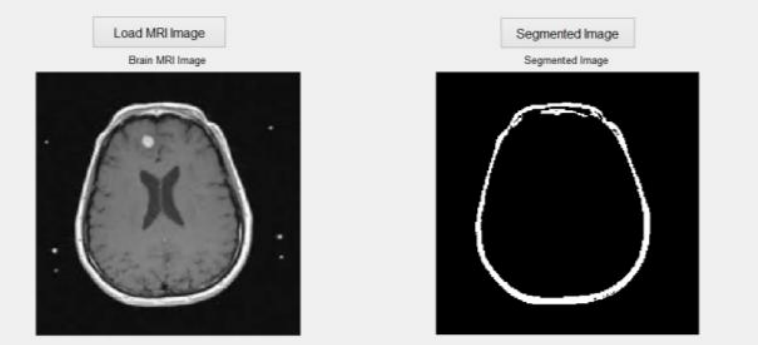

Fig 5 MRI Segmented Image having Tumor at the Centre

\section{CONCLUSIONS}

It is very useful to detect flaws in industrial application to improve the quality and reduce the cost of product. The image obtained after the implementation of region growing technique produced high quality segmentation on the wide range of gray scale of various biomedical modality images. It can easily be adapted to different image applications by substituting the suitable feature measure. By using regiongrowing technique the size and position of tumors can be depicted more accurately

A limitation of this algorithm is that the threshold value has to be judged by the medical expert, based on histogram of the image. Further development i.e. next algorithm will be comparison of all segmentation techniques with Fuzzy Segmentation

\section{REFERENCES}

[1] Ullrich kothe "Primary image segmentation"

[2] H. Jiang, J.Toriwaki and H.Suzuki "comperitive performance evaluation of segmentation method based on region growing and division" Syst. Compute. Jpn,Vol24,No 13, pp28-42,1993

[3] N.R.Pal and S.K.Pal " A review on segmentation technique”, pattern recognit., Vol 26, pp1277-1294, 1993

[4] P.L.Palmer,H.Dabis, and J.Kittler," A performance measure for boundry detection algorithm," Comput. Vis. Image Understand, vol 63, pp.476-494, 1996

[5] Henstock and d. Chelberg, "Automatic Gradient Threshold Determination for edge detection," IEEE Trans. On Image Processing, vol. 5, no. 5, pp. 784-787, may 1996.
[6] F.Mclen and M.Jernigan, "Hierachical edge detection," Computer Vision Graphics, Image Processing, vol, 44, p 350-366, 1988.

[7] S. Venkatesh and L.J. Kitchen, "Edge evolution using necessary components," Computer Vision, Graphics, Image Processing: Graphic, Models Image Processing, vol.54, pp. 23-30, 1992.

[8] R. Henstock \& Chelberg "Automatic Gradient threshold determination for edge detection". IEEE Trans. on Image Processing, Vol 5, no.5, pp 784-787, May 1996

[9] "A threshold and region growing combined method for filament disappearance area detection in solar image" March 21-23, 2001 Jianlin Gas, Mingchu thole and Haimin wang, 2001 conference on information science and system, the Johns Hopkin university

[10] R. Adams and L. Bischof, "Seeded region growing," IEEE Trans. Pattern Anal. Machine Intell., Vol. 16 pp. 641-647, 1994

[11] A.J. Abrantes and J.S. Marques, " A class of constrained clustering algorithm for object boundary extraction," IEEE Trans. Image Processing, Vol. 5 pp. 1507-1521, 1996

[12] X. Yu and J. YiaJaaski, " A new algorithm for image segmentation based on region growing and edge detection," Proc. Int. Symp. Circuits and Systems, 1991, vol. 1, pp. 516-519.

[13] "Adaptive image region-growing" Yian-leng Chang and Xiaobo Li, IEEE Transaction on image processing, vol 3, no. 6, Nov 1994

[14] "Segmentation through variable-order surface fitting" by Besl and Jain, IEEE Transaction on Pattern Analysis and Machine Intelligence, Vol. 10, no.2, pp.167-192 1998.

[15] "Region growing" Mingyue Ding , Advanced image processing \& analysis, Jan 2004

[16] R. C. Gonzalez and R. E. Woods, "Digital Image Processing" Second edition 2002, Pearson Education Asia.

[17] Sonka M, Hlavac V, Boyle R. "Image Processing, Analysis, and Machine Vision" Second edition 2003. 